\title{
Body mass index and patient CT measurements as a predictor of benefit of intensity-modulated radiotherapy to the supraclavicular fossa
}

This article was published in the following Dove Press journal:

OncoTargets and Therapy

20 November 2013

Number of times this article has been viewed

\section{Vicky Koh' \\ Johann I Tang' \\ Bok Ai Choo' \\ Chek Wee Tan' \\ Boon Keat Lim² \\ Liang Shen ${ }^{3}$ \\ Jiade Jay $\mathrm{Lu}^{\prime}$}

'Department of Radiation Oncology, National University Cancer

Institute, Singapore; ${ }^{2}$ Department of Diagnostic Radiology, Khoo Teck Puat Hospital, Singapore; ${ }^{3}$ Department of Biostatistics, National University of Singapore, Singapore
Correspondence: Jiade Jay Lu Department of Radiation Oncology, National University Cancer Institute, Singapore, IE Kent Ridge Road, NUHS Tower Block, Level 7, Singapore II 9228 Tel +6598186358

Email mdcljj@nus.edu.sg
Background: Irradiation of the supraclavicular fossa is commonly used as part of adjuvant breast radiotherapy. Intensity-modulated radiotherapy (IMRT) may be used to target this region accurately, and there are subgroups of patients that may benefit more from IMRT than others. We identify the benefit of IMRT over fixed-depth dose prescription to the supraclavicular fossa in patients of different builds in a clinical setting.

Methods: Fifteen patients who received radiotherapy to the left breast and supraclavicular fossa were selected. Computed tomographic planning was used to generate plans for supraclavicular fossa coverage. Dose prescription to $1.5 \mathrm{~cm}$ and $3.0 \mathrm{~cm}$ depths was compared with IMRT plans. Coverage of the planning target volume and dose to the organs at risk were compared and correlated with patient body mass index (BMI) and computed tomography measurements.

Results: Within the IMRT group, increasing depth of the supraclavicular fossa produced significantly better coverage of the planning target volume with IMRT. IMRT resulted in lower mean doses to the brachial plexus $(P=0.00)$ when compared with $1.5 \mathrm{~cm}$ and $3 \mathrm{~cm}$ depth dose prescriptions, but higher maximum brachial plexus doses. IMRT was more beneficial in patients with lower BMI because this resulted in a decreased maximum brachial plexus dose ( $P$-values of 0.03 and 0.001 when compared with $1.5 \mathrm{~cm}$ and $3.0 \mathrm{~cm}$ depth dose prescriptions, respectively). Higher patient BMI resulted in a lower dose contribution of IMRT to the cord ( $P$-values 0.066 and 0.034 when compared with $1.5 \mathrm{~cm}$ and $3.0 \mathrm{~cm}$ depth dose prescriptions respectively).

Conclusion: IMRT of the supraclavicular fossa results in lower brachial plexus doses for patients with low BMI while patients with higher BMI benefit from lower mean cord doses. IMRT provides superior coverage of the planning target volume, especially in patients with a deeper supraclavicular fossa.

Keywords: intensity-modulated radiotherapy, radiotherapy, breast cancer, supraclavicular fossa

\section{Introduction}

Recent evidence has shown the benefit of regional nodal irradiation in the setting of breast conservation therapy and for patients with one to three involved lymph nodes. ${ }^{1,2}$ Irradiation of the supraclavicular fossa is generally offered to patients with breast tumors $5 \mathrm{~cm}$ or greater in size and/or with four or more axillary lymph nodes positive for metastases found on axillary dissection and premenopausal women with any number of positive lymph nodes. This strategy has avoided excessive irradiation of the axilla and aims to improve therapeutic ratio by focusing on the undissected regions at higher risk of recurrence, while limiting the risk of lymphedema, brachial plexopathy, and lung fibrosis by excluding nodal areas that have undergone surgery. 
Most radiation oncologists treat the breast and supraclavicular lymph nodes with a conventional threefield approach. ${ }^{3}$ However, this has been shown to be dosimetrically inadequate. Coverage of the supraclavicular fossa has been poor, and this approach leads to high doses to the organs at risk. ${ }^{4}$

The supraclavicular lymph nodes have been found to vary widely in different patients. While deeper supraclavicular fossae are related to a larger anterior-posterior diameter in a linear relationship, lymph node depth varies between one-fifth and one half of the anterior-posterior diameter. ${ }^{5}$ Liengsawangwong et al $1^{4}$ compared optimized computed tomography (CT) planning of the supraclavicular fossa with fixed-depth dose prescription in patients of varying body mass index (BMI) and showed that optimized CT planning provided the best coverage for all patients over fixed-depth dose prescription across patients of normal, overweight, and obese BMI. BMI was also found to be significantly correlated with depth of the supraclavicular fossa. In addition, treatment of the supraclavicular fossa is also challenging due to its irregular shape, variability between patients, and the many organs at risk that either traverse it or are in close proximity to it.

Intensity-modulated radiotherapy (IMRT) has been used in various studies to improve coverage of the supraclavicular fossa, but this technique has been at the expense of increased doses to the lung, spinal cord, and other organs at risk., However, there may be subgroups of patients of certain build or measurements that would have greater benefit from IMRT. It is important to identify these groups of patients as we increasingly embrace IMRT as a modality for adjuvant breast irradiation, given its superior target coverage and increasing ease of delivery with newer machines.

In this study, we look at the benefit of inverse planning IMRT over fixed-depth dose prescription in improving coverage and reducing the dose to organs at risk for patients with different BMIs and of different builds to find a group that would benefit most from planning of IMRT to the supraclavicular fossa.

\section{Materials and methods}

This study was conducted between 2009 and 2011 and was approved by the institutional review board at the National University Hospital, Singapore (DSRB 2009/00640). Fifteen consecutive patients who had completed radiotherapy to the left breast and supraclavicular fossa in 2009 were selected for this planning study. The CT images of these patients were planned using tangents for the breast tissue matched superiorly with a single anterior oblique field prescribed to a depth of $1.5 \mathrm{~cm}$ and $3.0 \mathrm{~cm}$ to cover the supraclavicular nodes. These were compared with the IMRT technique (inverse planning) for supraclavicular nodal coverage.

\section{CT simulation}

The patients were simulated supine with both arms overhead and head turned to the contralateral side of treatment. The superior, inferior, and mid axillary borders were marked with fiducials and the patient's mid line was defined by placement of a radio-opaque wire over the sternum. $\mathrm{CT}$ images were obtained using a Brilliance Big Bore CT scanner (Philips, Eindhoven, the Netherlands) at $5 \mathrm{~mm}$ slices through the region of interest. Four-dimensional CT scanning was not used in this study.

\section{Structure delineation}

Supraclavicular lymph node regions, breast tissue, tumor bed, brachial plexus, spinal cord, and ipsilateral lung and heart were manually contoured by the same physician (VK) and reviewed with a radiologist (BKL). Breast volume and nodal volume definitions from the Radiation Therapy Oncology Group breast contouring atlas were used. The depth of the supraclavicular nodes was defined as the deepest portion of the subclavian artery and vein at that level while the anterior-posterior diameter was measured at the location of the supraclavicular nodes as described by Bentel et al. ${ }^{5}$

The supraclavicular fossa was delineated according to published imaging guidelines: the borders are defined medially by the lateral edge of the trachea excluding the thyroid gland, superiorly by the inferior edge of the cricoid cartilage, anteriorly by the posterior border of the sternocleidomastoid muscle, posterolaterally by the anterior border of the anterior scalene muscle, and posteromedially to include the carotid artery and jugular vein. At the inferior aspect of the supraclavicular fossa, the anterior border of the subclavian vessels forms the posterior border of the fossa.

\section{Definition of clinical and planning target volumes}

The supraclavicular nodal clinical target volume was defined as above. This was expanded uniformly by $0.5 \mathrm{~cm}$ to create a regional nodal planning target volume which excluded the superficial overlying skin.

\section{Dosimetric techniques}

All plans were generated by the same planner (CWT) using Xio Treatment Planning system version 6.4 (Elekta, 
Stockholm, Sweden). For conventional treatment, a monoisocentric technique was used to target the lymph nodes and ipsilateral breast. A total dose of $50 \mathrm{~Gy}$ in 25 fractions planned with $6 \mathrm{MV}$ or $10 \mathrm{MV}$ photons was prescribed to the breast and regional lymph nodes. A single anterior supraclavicular field angled 10 degrees off cord was planned to match the superior border of the tangential fields. Two plans were generated: one with the dose prescribed to $1.5 \mathrm{~cm}$ using $6 \mathrm{MV}$ and another with the dose prescribed to $3.0 \mathrm{~cm}$ using $10 \mathrm{MV}$ at the level of the supraclavicular nodes. The borders of the supraclavicular field were defined medially at the mid line, laterally at the lateral aspect of the coracoid process, superiorly at the lower edge of the thyroid cartilage, and inferiorly matched to the tangential fields.

Inverse planning for IMRT was used. Coverage of the planning target volume required five IMRT fields including one posterior oblique field. Beam angles were chosen to minimize the exit dose to the contralateral lung. Spinal cord inclusion within the IMRT field was allowed.

The planning objectives were to provide $95 \%$ coverage for the planning target volume and to minimize the dose to the contralateral lung and the spinal cord. The following dose parameters were used for the IMRT algorithm: planning target volume Dmax 52 Gy, ipsilateral lung V20 < 30 Gy, spinal cord +5 mm Dmax 45 Gy, brachial plexus Dmax 50 Gy, and heart V20 < 30 Gy. The Dmin for the planning target volume was set higher than the prescription dose to improve target coverage in the final plan. All fields were planned with a single isocenter setup in which a physical match between the tangential and nodal fields is maintained with half beam blocking. Tangential and nodal IMRT elements undergo IMRT optimization simultaneously with fixed jaws. Representative isodose distributions from all the planning techniques are shown in Figure 1.

\section{Statistical analysis}

The coverage of each technique with inverse planned IMRT for targets and doses to organs at risk was summarized descriptively. Dose volume histograms were used to determine doses to targets and normal structures. The coverage for targets and doses to organs at risk was compared between different techniques with inverse planned IMRT by using the paired $t$-test.

Spearman's correlation coefficient was used to measure the strength of correlation of BMI, anterior-posterior diameters, and supraclavicular fossa depths with the increase in coverage for targets and decrease of doses to organs at risk using IMRT compared with the dose prescribed

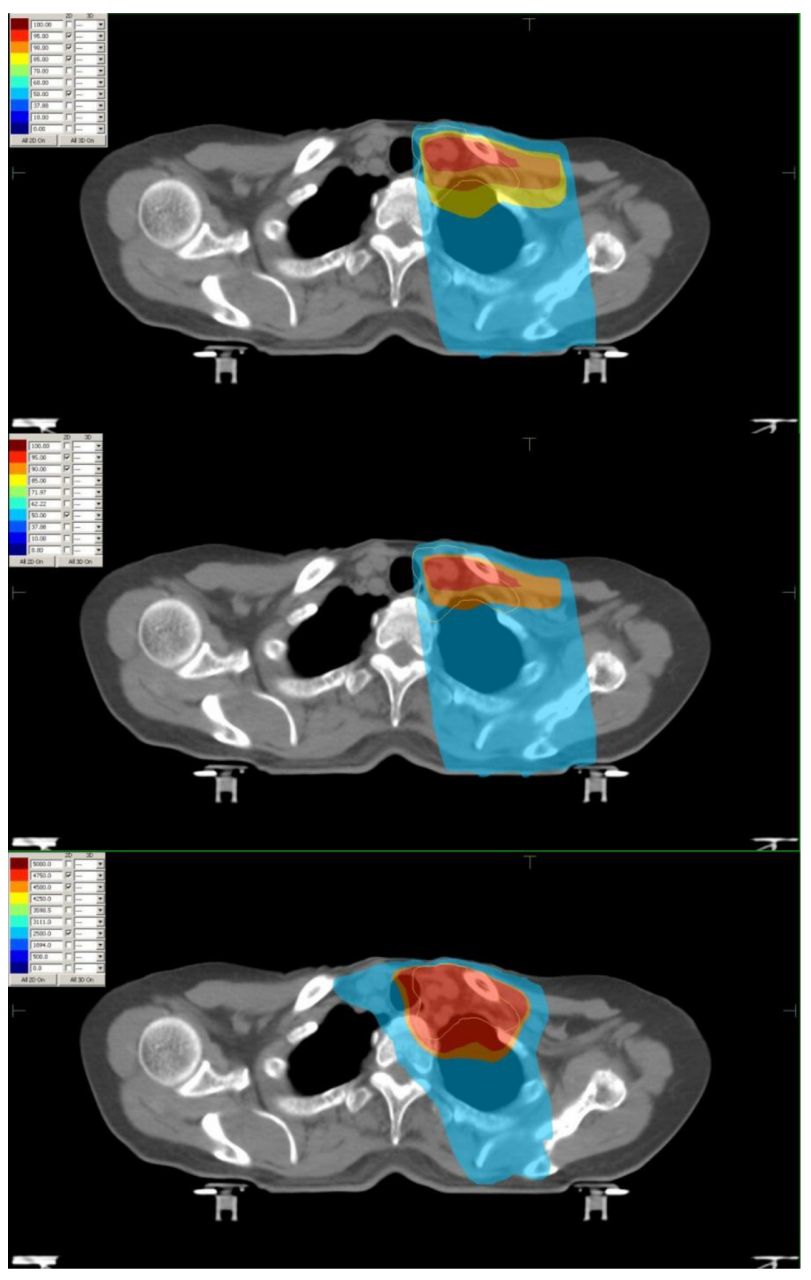

Figure I Dose distribution of $1.5 \mathrm{~cm}$ and $3.0 \mathrm{~cm}$ depth dose prescriptions and intensity modulated radiotherapy.

to a fixed depth. For all analyses, a $P$-value $<0.05$ was considered to be statistically significant.

\section{Results}

\section{Patient characteristics}

The patients were aged 45-57 years with stage II and III intraductal carcinoma involving the left breast that required adjuvant radiotherapy to the breast and supraclavicular lymph nodes. The BMIs of the patients ranged from 13.2 to 30 (mean 23.5, median 25). BMI was calculated by dividing the patient's mass in kilograms as recorded on the day of CT simulation by the square of the patient's height in meters.

\section{Planning target volume coverage}

When compared with $1.5 \mathrm{~cm}$ and $3 \mathrm{~cm}$ depth dose prescription, we found superior planning target volume coverage with IMRT ( $P$-value $<0.001$, Figure 2 ). In the IMRT group, increasing depth of the supraclavicular fossa was positively 
correlated with better coverage of the planning target volume with IMRT ( $P$-value 0.041 , correlation coefficient 0.533 ). However, as the patient's BMI or anterior-posterior diameter increased, we found no improvement of planning target volume coverage in the IMRT group $(P$-values 0.461 and 0.158 , respectively).

\section{Organs at risk}

IMRT resulted in lower mean doses to the brachial plexus $(P$-values 0.00$)$ when compared with $1.5 \mathrm{~cm}$ and $3 \mathrm{~cm}$ depth dose prescription, but higher maximum brachial plexus doses (Table 1). The average maximum dose to the brachial plexus was 5,383 cGy in the IMRT group compared with $4,910 \mathrm{cGy}$ and 5,148 cGy in the $1.5 \mathrm{~cm}$ and $3.0 \mathrm{~cm}$ depth dose prescription groups, respectively. Increasing BMI, supraclavicular fossa depth, and anterior-posterior diameter were also found to be associated with increasing maximum dose to the brachial plexus with the use of IMRT (Table 2). IMRT resulted in higher maximum lung doses and lower mean lung doses compared with $1.5 \mathrm{~cm}$ and $3.0 \mathrm{~cm}$ depth dose prescription.

IMRT plans also had a higher mean and maximum cord dose compared with fixed-dose prescription. The mean cord dose was $806 \mathrm{cGy}$ with IMRT compared with $102 \mathrm{cGy}$ and $120 \mathrm{cGy}$ for $1.5 \mathrm{~cm}$ and $3.0 \mathrm{~cm}$ depth dose prescription, respectively. Patients with higher BMI and anterior-posterior diameter had lower mean cord doses with the use of IMRT compared with those with higher BMI and anterior-posterior diameter. There was no significant difference between the different techniques with regard to heart dose.

\section{$\mathrm{BMI}$}

When we analyzed BMI over a range, we found that as the BMI of the patient decreased, the benefit of IMRT over fixed-depth dose prescription improved, as evidenced by decreasing maximum brachial plexus dose ( $P$-values 0.03 and 0.001 for $1.5 \mathrm{~cm}$ and $3.0 \mathrm{~cm}$ prescription, respectively). As the BMI of the patient increased, there was lower dose contribution of IMRT to the cord when compared with $1.5 \mathrm{~cm}$ and $3.0 \mathrm{~cm}$ depth dose prescription ( $P$-values 0.066 and 0.034 , respectively).

\section{Other CT measurements}

Within the IMRT group, patients with a deeper supraclavicular fossa benefited most from improved planning target volume coverage. Patients with smaller anterior-posterior diameters benefited from lower mean cord doses and lower maximum brachial plexus doses when IMRT was used $(P$-values 0.04 and 0.01 when compared with $1.5 \mathrm{~cm}$ and $3.0 \mathrm{~cm}$ depth dose prescription, respectively). We found a linear relationship between depth of the supraclavicular fossa and BMI in our study group.

\section{Discussion}

Our study showed superior coverage of the planning target volume and lower mean doses to the brachial plexus and ipsilateral lung with the use of IMRT but at the expense of a higher dose to the cord and a higher maximum dose to the brachial plexus and lung. In addition, we found that patients with lower BMI benefited from IMRT with lower maximum brachial plexus doses. Patients with higher BMI had lower mean cord doses. Patients with a deeper supraclavicular fossa benefited from significantly improved planning target volume coverage within the IMRT group.

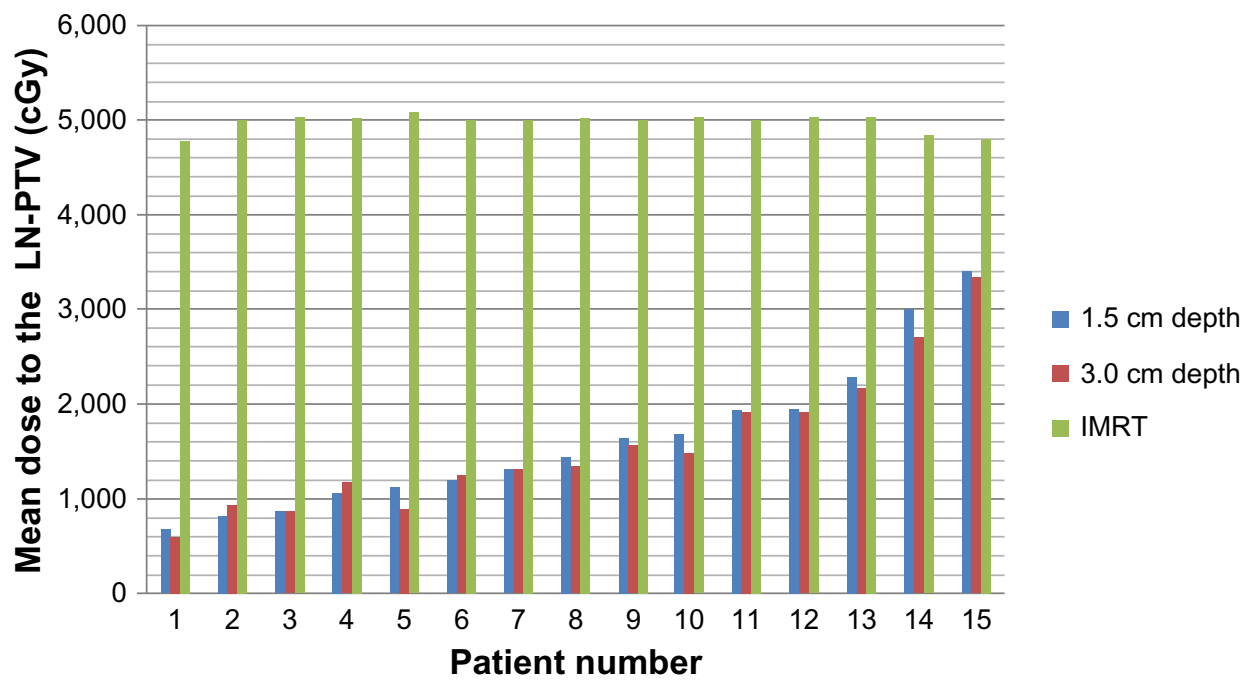

Figure 2 Coverage of the supraclavicular fossa planning target volume by intensity-modulated radiotherapy compared with fixed-depth dose prescription. Abbreviations: IMRT, intensity-modulated radiotherapy; PTV, planning target volume. 
Table I Comparison of results for each of the techniques used

\begin{tabular}{|c|c|c|c|c|c|}
\hline & \multicolumn{3}{|c|}{ Mean in cGray (standard deviation) } & \multicolumn{2}{|c|}{$P$-value (comparison with IMRT) } \\
\hline & $1.5 \mathrm{~cm}$ depth & $3 \mathrm{~cm}$ depth & IMRT & $1.5 \mathrm{~cm}$ depth & $3 \mathrm{~cm}$ depth \\
\hline PTV $95 \%$ & I,620 (789) & $\mathrm{I}, 403(728)$ & $4,974(92)$ & $<0.001$ & $<0.001$ \\
\hline Brachial plexus (mean dose) & $3,678(325)$ & $3,876(303)$ & $2,774(400)$ & $<0.001$ & $<0.001$ \\
\hline Brachial plexus (maximum dose) & $4,910(179)$ & $5,148(230)$ & $5,383(132)$ & $<0.001$ & 0.002 \\
\hline Heart (mean dose) & $3.80(1.82)$ & $4.60(3.96)$ & $4.00(3.05)$ & 0.715 & 0.508 \\
\hline Heart (maximum dose) & $22.6(8.68)$ & $25.1(15.2)$ & $23.4(13.1)$ & 0.695 & 0.668 \\
\hline Cord (mean dose) & $102(58.3)$ & $120(8 \mid .4)$ & $806(440)$ & $<0.001$ & $<0.001$ \\
\hline Cord (maximum dose) & $1,320(506)$ & $\mathrm{I}, 430(504)$ & $3,846(299)$ & $<0.001$ & $<0.001$ \\
\hline Left lung (mean dose) & $443(84.8)$ & $469(95.2)$ & $400(\mathrm{III})$ & 0.11 & 0.014 \\
\hline Left lung (maximum dose) & $4,814(192)$ & $5,059(259)$ & $5,42 \mid(\mid 13)$ & $<0.001$ & $<0.001$ \\
\hline
\end{tabular}

Note: The Bonferroni technique was used for multiple comparisons.

Abbreviations: IMRT, intensity-modulated radiotherapy; PTV, planning target volume.

While IMRT has been shown in other studies ${ }^{4,7-9}$ to be superior to fixed-depth dose prescription in coverage of the supraclavicular fossa in many patients, this is the first study, to the authors' knowledge, that identifies specific benefits for patients of different BMI, supraclavicular fossa depth, and anterior-posterior diameter. Our results show that patients who will benefit more from IMRT to the supraclavicular region can be identified in the clinic by measurement of BMI. In addition, measurements such as depth of the supraclavicular fossa can assist the clinician in selection of technique in the planning stage of radiotherapy in most busy centers.

In this study, we found that IMRT significantly decreased the mean dose to the brachial plexus over fixed-depth dose prescription. The consensus recommendations on brachial plexus dose tolerance by Emami et al ${ }^{10}$ considered the brachial plexus and the cauda equine together and suggested a value for a $5 \%$ risk at 5 years of 62,61 , and 60 Gy and a value for a $50 \%$ risk at 5 years of 77,76 , and 75 Gy for one third, two thirds, and the whole organ, respectively. There are, however, multiple reports of brachial plexopathy in breast cancer patients, mainly occurring in those who received treatment using cobalt sources or using techniques in which overlap of radiotherapy fields occurred resulting in high doses per fraction being delivered. ${ }^{11-13}$ While even the maximum doses to the brachial plexus reached in adjuvant treatment of the supraclavicular fossa today would not be high enough to cause plexopathy based on the Emami data, the decrease in mean dose to the brachial plexus with the use of IMRT is helpful for treatment of gross nodal disease in the supraclavicular fossa where doses of $60 \mathrm{~Gy}$ and above would be required.

Previous studies of CT-optimized plans or IMRT to treat the supraclavicular region have shown benefit in planning target volume coverage at the expense of organs at risk, ${ }^{4,9,10}$ such as the lung and cord. We had similar findings using our IMRT technique. Our technique also did not find any significant benefit to the heart dose with IMRT. We also confirmed a linear relationship between depth of the supraclavicular fossa and BMI in our patient group, which has been observed in other studies. ${ }^{4}$

However, some IMRT studies included the breast tissue as part of the IMRT planning target volume or as a separate volume with a matchline between the lymph node and breast

Table 2 P-values when fixed-depth dose prescription is compared with IMRT across increasing AP diameter, SCF depth, and BMI

\begin{tabular}{|c|c|c|c|c|c|c|}
\hline & \multicolumn{2}{|l|}{ AP diameter } & \multicolumn{2}{|l|}{ SCF depth } & \multicolumn{2}{|l|}{ BMI } \\
\hline & $1.5 \mathrm{~cm}$ depth & $3.0 \mathrm{~cm}$ depth & $1.5 \mathrm{~cm}$ depth & $3.0 \mathrm{~cm}$ depth & $1.5 \mathrm{~cm}$ depth & $3.0 \mathrm{~cm}$ depth \\
\hline PTV 95\% & 0.685 & 0.062 & 0.879 & 0.196 & 0.817 & 0.302 \\
\hline Brachial plexus (mean dose) & 0.742 & 0.800 & 0.125 & 0.114 & 0.508 & 0.438 \\
\hline Brachial plexus (maximum dose) & $0.004 *$ & $0.001 *$ & $0.044 *$ & $0.012^{*}$ & $0.030 *$ & $0.001 *$ \\
\hline Heart (mean dose) & 0.817 & 0.939 & 0.898 & 0.842 & 0.780 & 0.875 \\
\hline Heart (maximum dose) & 0.960 & 0.340 & 0.270 & 0.914 & 0.844 & 0.406 \\
\hline Cord (mean dose) & $0.046 *$ & $0.028 *$ & 0.383 & 0.341 & 0.066 & $0.034^{*}$ \\
\hline Cord (maximum dose) & 0.830 & 0.945 & 0.960 & 0.924 & 0.345 & 0.440 \\
\hline Left lung (mean dose) & 0.668 & 0.578 & 0.067 & 0.090 & 0.898 & 0.678 \\
\hline Left lung (maximum dose) & $0.029 *$ & 0.132 & $0.017 *$ & 0.105 & 0.201 & 0.336 \\
\hline
\end{tabular}

Note: *Significant $P$-values.

Abbreviations: AP, anterior-posterior; BMI, body mass index; SCF, supraclavicular fossa; IMRT, intensity-modulated radiotherapy; PTV, planning target volume. 
IMRT planning target volumes. We were concerned about dose contribution from the IMRT breast plan that would affect the results. Given that we were studying techniques specific to the supraclavicular fossa and to remove confounders from the breast plan, such as different breast sizes in different patients, we chose to match the lower border of the supraclavicular fossa field with tangents. This was useful in achieving our study goals and allowed the option of adding IMRT breast fields should the tangents produce an inferior plan. Its ease of use also made this a practical and effective way of planning treatment.

The shortcomings of this study include its small sample size. Some of the $P$-values we obtained were borderline significant for some organs at risk which showed only a mild correlation. A larger sample size would have made the correlations stronger or shown no correlation.

Also, while we were able to decrease the mean brachial plexus and lung doses, we were unable to decrease the maximum doses to these organs at risk with the use of IMRT. The absolute maximum dose to the brachial plexus in our study group was 5,661 cGy, 4,271 cGy for cord, and 5,615 cGy for the lung. While these doses are below tolerance doses, future techniques used for planning and treatment should try to decrease the maximum doses to these organs, especially the cord and brachial plexus.

Possible questions that need to be answered in future studies would be the use of different modalities such as proton beam therapy and helical tomotherapy to treat the supraclavicular fossa. The long-term toxicity of the different treatment techniques could also be explored in randomized studies. The Z0011 study ${ }^{14}$ suggests that tangential fields may be sufficient adjuvant therapy for early-stage breast cancer with favorable risk factors, suggesting that the tangential fields adequately cover some axillary lymph node groups precluding the need for an axillary dissection. It is possible that this coverage may vary according to BMI and build as well. Future studies should look again at traditional tangential field coverage in relation to build and correlate this with overall survival.

\section{Conclusion}

IMRT of the supraclavicular fossa results in lower brachial plexus doses for patients with low BMI while patients with higher BMI benefit from lower mean cord doses. IMRT

OncoTargets and Therapy

\section{Publish your work in this journal}

OncoTargets and Therapy is an international, peer-reviewed, open access journal focusing on the pathological basis of all cancers, potential targets for therapy and treatment protocols employed to improve the management of cancer patients. The journal also focuses on the impact of management programs and new therapeutic agents and protocols on

Submit your manuscript here: http://www.dovepress.com/oncotargets-and-therapy-journal provides superior coverage of the planning target volume, especially in patients with deeper supraclavicular fossa.

\section{Disclosure}

The authors report no conflicts of interest in this work.

\section{References}

1. Whelan TJ, Olivotto I, Ackerman J, et al. NCIC-CTG MA 20: an Intergroup trial of regional nodal irradiation in early breast cancer. J Clin Oncol. 2011;29:779s.

2. Truong PT, Jones SO, Kader HA, et al. Patients with $\mathrm{t} 1$ and $\mathrm{t} 2$ breast cancer with one to three positive nodes have higher local and regional recurrence risks compared with node negative patients after breastconserving surgery and whole-breast radiotherapy. Int $J$ Radiat Oncol Biol Phys. 2009;73:357-364

3. Pierce LJ, Moughan J, White J, et al. 1998-1999 patterns of care study process survey of national practice patterns using breast-conserving surgery and radiotherapy in the management of stage I-II breast cancer. Int J Radiat Oncol Biol Phys. 2005;62:183-192.

4. Liengsawangwong R, Yu TK, Tzouh-Liang S, et al. Treatment optimization using computed tomography-delineated targets should be used for supraclavicular irradiation for breast cancer. Int $J$ Radiat Oncol Biol Phys. 2007;69:711-715.

5. Bentel GC, Marks LB, Hardenbergh PH, et al. Variability of the depth of supraclavicular and axillary lymph nodes in patients with breast cancer: is a posterior axillary boost field necessary? Int J Radiat Oncol Biol Phys. 2000;47:755-758.

6. Sethi RA, Hyun SN, Gabor J, et al. Comparison of three-dimensional versus intensity-modulated radiotherapy techniques to treat breast and axillary level III and supraclavicular nodes in a prone versus supine position. Radiother Oncol. 2012;102:74-81.

7. Lomax A, Cella L, Weber D, et al. Potential role of intensity modulated photons and protons in the treatment of the breast and regional nodes. Int J Radiat Oncol Biol Phys. 2003;55:785-792.

8. Cavey ML, Bayouth JE, Endres EJ, et al. Dosimetric comparison of conventional and forward-planned intensity-modulated techniques for comprehensive locoregional irradiation of post-mastectomy left breast cancers. Med Dosim. 2005;30:107-116.

9. Dogan N, Cuttino L, Lloyd R, et al. Optimised dose coverage of regional lymph nodes in breast cancer: the role of intensity-modulated radiotherapy. Int J Radiat Oncol Biol Phys. 2007;68:1238-1250.

10. Emami B, Lyman J, Brown A, et al. Tolerance of normal tissue to therapeutic irradiation. Int J Radiat Oncol Biol Phys. 1991;21: 109-122.

11. Basso-Ricci S, della Costa C, Viganotti G, et al. Report on 42 cases of post-irradiation lesions of the brachial plexus and their treatment. Tumori. 1980;66:117-122.

12. Johansson S, Svensson H, Denekamp J. Timescale of evolution of late radiation injury after postoperative radiotherapy of breast cancer patients. Int J Radiat Oncol Biol Phys. 2000;48:745-750.

13. Powell S, Crooke J, Parsons C. Radiation-induced brachial plexus injury: follow-up of two different fractionation schedules. Radiother Oncol. 1990;18:213-220.

14. Giuliano A, Hunt K, Ballman K. Axillary dissection vs no axillary dissection in women with invasive breast cancer and sentinel node metastasis. JAMA. 2011;305:569-575.

\section{Dovepress}

patient perspectives such as quality of life, adherence and satisfaction The manuscript management system is completely online and includes a very quick and fair peer-review system, which is all easy to use. Visit http://www.dovepress.com/testimonials.php to read real quotes from published authors. 